\title{
IS THERE OBSERVATIONAL EVIDENCE FOR THE EVAPORATION OF THE INNER ACCRETION DISK IN DWARF NOVAE AT QUIESCENCE?
}

\author{
C. LA DOUS ${ }^{1}$, E. MEYER-HOFMEISTER ${ }^{2}$, F. MEYER ${ }^{2}$ \\ 1.ESA/IUE Observatory, Villafranca del Castillo, Apartado \\ 50727, 28080 Madrid, Spain \\ 2.Max-Planck-Institut für Astrophysik, \\ Karl-Schwarzschild-Str. 1, 85740 Garching, Germany
}

\begin{abstract}
We present the preliminary results of a comparison of observed general patterns of quiescent dwarf novae and theoretical predictions of the disk evaporation model. We demonstrate that, even though details still have to be clarified, on the whole there is strong evidence that observations and theory are in agreement.
\end{abstract}

\section{Introduction}

The observations of dwarf novae in quiescence and during rise to outburst provide some features which seem to be in contradiction to the standard theoretical modelling of dwarf nova outbursts, where mass is accumulated during quiescence until the instability causes the next outburst.

(i) The angle dependence of the equivalent widths of UV emission lines: at low inclinations all lines are strongly in emission; as the inclination increases, the equivalent width decreases and at angles larger than some $60^{\circ}$ or $70^{\circ}$ only an essentially featureless continuum can be seen ${ }^{1}$ (la Dous 1991). From this it can be concluded that there is an optically thin emission source radiating at UV wavelengths and that this source is predominantly confined to the orbital plane of the system.

(ii) The white dwarfs: it has been demonstrated that on the basis of the SWP spectrum alone it is possible to roughly determine the temperature of the white dwarf in quiescent dwarf novae (la Dous 1996a,

\footnotetext{
${ }^{1}$ Except for the Ly $\alpha$ absorption line which is strong in some objects; we will deal with this in the next section.
} 
1996b; Hassall \& la Dous 1996). The white dwarf spectrum then can be subtracted from the observed integral spectrum. What remains is interpreted as the spectrum of the quiescent accretion disk. To zeroth approximation this can be fitted with a single blackbody having a temperature between 9000 and $15000 \mathrm{~K}$. In all objects for which more than one quiescent spectrum is available, the flux level is not constant with time, but changes are consistent with the assumption that they are entirely due to a temperature change in the central object.

(iii) In many dwarf novae a sometimes pronounced delay in the rise to outburst between optical and UV wavelengths radiation can be observed. It is too long to be explained by the spreading of the hot state in the disk. It seems that the inner disk first has to be filled with material before it can go into outburst (Meyer \& Meyer-Hofmeister 1989).

(iv) In a cool accretion disk mass flows with a rate of $10^{-13} \ldots 10^{-12} \mathrm{M}_{\odot} \mathrm{yr}^{-1}$ towards the white dwarf. This is at least an order of magnitude too low for explaining the actually observed X-ray and UV fluxes at quiescence, which require rates on the order of $10^{-11} \mathrm{M}_{\odot} \mathrm{yr}^{-1}$ (e.g. van der Woerd \& Heise 1987; Szkody et al. 1991).

\section{The evaporation model}

All these features can be understood within the framework of the disk evaporation model (Meyer \& Meyer-Hofmeister 1994; Liu, Meyer \& MeyerHofmeister 1995). The interaction of a hot corona with the underlying cool disk in dwarf nova systems leads to an evaporation of the inner disk around the white dwarf. In contact with the corona, material evaporates at higher gravitational potential and accretes on the compact star at lower gravitational potential. Due to angular momentum this mass flow takes the form of a coronal accretion disk, which is heated by friction. The process works best close to the white dwarf, thus evaporating the innermost part of the disk first. As a consequence this hole has to be filled up on the diffusion time-scale during the next outburst causing a delay of radiation from the innermost disk, that is, of UV flux.

The evaporation model gives typical mass flow rates of about $10^{-11}$ to $310^{-11} \mathrm{M}_{\odot} \mathrm{yr}^{-1}$. Part of it leaves the system as a very hot wind, the remaining part is accreted onto the white dwarf. X-rays are produced mainly in the optically thin thermal conductive boundary layers above the accretion disk (a small fraction) and the white dwarf (main part of the radiation). The less hot regions below are heated, so that the UV radiation originates from both the disk and the white dwarf, whereby the latter varies its surface temperature depending on the accretion rate. 

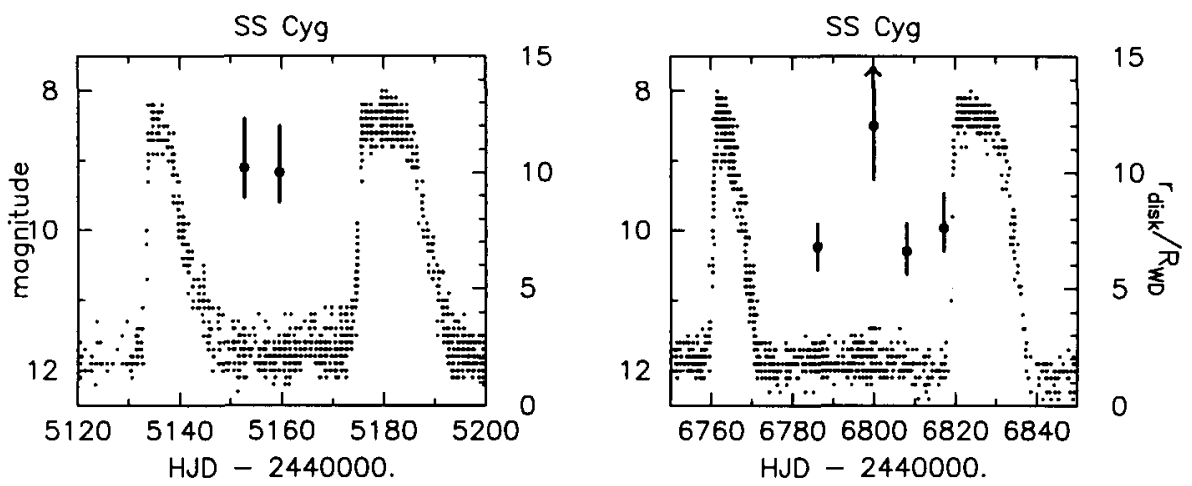

Figure 1. Variations of the inner disk radius during the course of two quiescent intervals of SS Cyg. The error bars shown are those resulting from uncertainties of $\pm 1000 \mathrm{~K}$ in the temperature of the optically thick accretion disk. The photometric data are courtesy of the AAVSO.

\section{Interpretation of observations}

The ratio of fluxes from the white dwarf and the disk provide information on the size of the disk area where most of the radiation is emitted. Taking blackbody radiation as a first approximation, the ratio of the fluxes is related to the size of the emitting areas and, therefore, to the ratio of the square of the radii. For assumed inclination, and mass and radius of white dwarf we derive the disk radius near the inner edge where most radiation comes from. If we further assume that the cool underlying disk region is heated by the coronal layers above, we can derive the evaporation rate (= mass flow rate in the corona). From the observational side the necessary inputs are the fluxes and temperatures of white dwarf and accretion disk, which can be derived directly from UV spectra. The most delicate point, and at the same time the most difficult one, is the determination of the temperature of the optically thick accretion disk.

Neglecting the effect of mass accumulation in the disk, theory would predict a progressive evaporation of the accretion disk during a quiescent state and, consequently, a monotonic increase of the inner disk radius. Analyzing sequences of spectra taken during the same quiescent intervals of SS Cyg, we find that this is not the case, that the inner disk radius varies non-monotonically (Fig. 1). This behaviour might be due to the heating of the disk by the corona above and the material flowing inward from outer regions. The derived radii, of order 5 to 10 white dwarf radii and evaluated coronal mass flow rates of about $10^{-11} \mathrm{M}_{\odot} \mathrm{yr}^{-1}$ confirm the basic predictions of the evaporation model. For details we refer to a forthcoming publication (la Dous, Meyer-Hofmeister \& Meyer 1996). 


\section{References}

Hassall, B.J.M., la Dous, C., 1996, these proceedings, p85

la Dous, C., 1991, A\&A, 252, 100

la Dous, C., 1996a, in preparation

la Dous, C., 1996b, A\&A, in preparation

la Dous, C., Meyer-Hofmeister, E., Meyer, F., 1996, A\&A, in preparation

Liu, F.K., Meyer, F., Meyer-Hofmeister, E., 1995, A\&A, 300, 823

Meyer, F., Meyer-Hofmeister, E., 1989, A\&A, 221, 36

Meyer, F., Meyer-Hofmeister, E., 1994, A\&A, 288, 175

Szkody, P., Mattei, J.A., Waagen, E.O., Staeblein, C., 1991, Ap. J. Supp., 76, 359

van der Woerd, H., Heise, J., 1987, MNRAS, 225, 141 